\title{
Vasorreatividade Coronariana após Reabsorção Total do Absorb BVS no Seguimento de 5 Anos
}

\author{
Coronary Vasoreactivity after Complete Bioresorption of Absorb BVS at 5-Year Follow-Up
}

\author{
Luis Renier Goncalves-Ramírez, ${ }^{1}$ Hipólito Gutíerrez, ${ }^{2}$ Fabián Julca, ${ }^{2}$ Maximiliano Germán Amado Escañuela, ${ }^{3}$ \\ Gretel Varvaro, ${ }^{4}$ Ignacio Amat-Santos ${ }^{2}$ \\ Hospital de León - Cardiologia, ${ }^{1}$ León - Espanha \\ Hospital Clinico Universitario de Valladolid, ${ }^{2}$ Castilla y León - Espanha \\ Hospital General de Segovia, ${ }^{3}$ Castilla y León - Espanha \\ Hospital General de Palencia Río Carrión, ${ }^{4}$ Castilla y León - Espanha
}

\section{Introdução}

Os suportes coronarianos bioabsorvíveis foram projetados para prevenir complicações em longo prazo relacionadas ao implante permanente de stents metálicos. O suporte vascular bioabsorvível eluidor de everolimus (Absorb BVS; Abbott Vascular, Santa Clara, Califórnia) foi um dos primeiros suportes vasculares bioabsorvíveis (BVS, do inglês bioresorbable vascular scaffold) a ser desenvolvido. O BVS Absorb é uma estrutura feita de ácido poli-L-láctico revestido com polímero poli-DL-láctico, que elui o fármaco antiproliferativo everolimus. ${ }^{1} \mathrm{O}$ BVS recebeu a marca CE para o tratamento de doença arterial coronariana em janeiro de 2011 e foi comercializado na maioria dos países europeus em 2012. ${ }^{2}$ Embora bons resultados tenham sido descritos inicialmente, ${ }^{3,4}$ estudos recentes têm questionado a segurança do dispositivo, sugerindo maior incidência de trombose e infarto do miocárdio. ${ }^{5,6}$ Além disso, a recuperação estrutural e funcional de segmentos coronários que receberam o suporte após a reabsorção do BVS não foi sistematicamente pesquisada em uma série consecutiva do mundo real. ${ }^{7}$ Descrevemos o caso de um paciente que foi avaliado por angiografia coronariana, tomografia de coerência óptica (TCO) e teste de vasorreatividade coronariana 5 anos após o implante do BVS.

\section{Relato de caso}

Um homem de 39 anos, ex-fumante, apresentou dor torácica atípica e teste de isquemia inconclusivo. A história pregressa incluía infarto do miocárdio com supradesnivelamento do segmento ST (IAMCSST) há 5 anos, relacionado a doença uniarterial tratada com um dispositivo

\section{Palavras-chave}

Implantes Absorviveis/normas; Intervenção Coronária Percutânea/métodos; Tecidos Suporte/normas; Doença Arterial Coronariana; Infarto do Miocárdio, Recuperação da Função Fisiológica.

Correspondência: Luis Renier Goncalves-Ramírez •

Hospital de León - Cardiologia - Altos de Navas León León 24071 - Espanha E-mail: luisrenier@hotmail.com

Artigo recebido em 07/11/2019, revisado em 22/01/2020, aceito em $22 / 01 / 2020$

DOI: https://doi.org/10.36660/abc.20190783
Absorb BVS de 3,5×28 mm no meio da artéria descendente anterior esquerda (ADA). No tempo presente, o paciente foi submetido a novo cateterismo coronário e não havia evidências de novas lesões ou reestenose. Em seguida, uma tomografia de coerência óptica (TCO) foi realizada sobre o segmento da ADA que recebeu o suporte, mostrando o dispositivo Absorb BVS totalmente reabsorvido, com o desenvolvimento de uma camada neoíntima bem organizada (Figura 1, Vídeo 1).

A vasorreatividade coronária foi avaliada com a administração de acetilcolina intracoronária. Bolus incrementais de acetilcolina foram infundidos $(2 \mu \mathrm{g}-20 \mu \mathrm{g}$ $100 \mu \mathrm{g}$ ) durante 3 minutos cada um, seguido de avaliação eletrocardiográfica, hemodinâmica, angiográfica e TCO da resposta funcional. Na dose máxima de acetilcolina, o paciente desenvolveu dor torácica e espasmo na ADA incluindo o segmento que recebeu o suporte - conforme observado por ambos, angiografia e TCO (Figura 2, Vídeo 2). Finalmente, um bolo intracoronário $(200 \mu \mathrm{g})$ de nitroglicerina foi administrado para aliviar o espasmo coronário e os sintomas. A repetição da angiografia e da TCO confirmou a resposta vasodilatadora.

\section{Discussão}

As tecnologias de BVS estão atualmente em destaque no mundo todo devido a uma taxa de eventos adversos de longo prazo maior do que a esperada e ao crescente questionamento quanto à reabsorção completa do dispositivo. ${ }^{8}$ Além disso, dados baseados em evidências de resultados funcionais em longo prazo dos vasos tratados com BVS ainda são escassos. ${ }^{9}$ De fato, se a vasomoção normal in vivo é recuperada ou não, permanece sem resposta.

Que seja de nosso conhecimento, este é o primeiro caso que mostra a recuperação morfológica e funcional de segmentos coronários que receberam suporte vascular, 5 anos depois da implantação do dispositivo Absorb BVS em um paciente da vida real. Como já foi descrito anteriormente, o Absorb BVS é finalmente reabsorvido pelo vaso 5 anos após sua implantação, com o desenvolvimento de uma camada rica em sinais visualizada por TCO no segmento que recebeu o suporte, o que corresponde à neoíntima e tecido subjacente, ${ }^{9,10}$ Por outro lado, a vasoconstrição paradoxal induzida pela acetilcolina e corrigida pela nitroglicerina adiciona informações específicas sobre a recuperação funcional das artérias coronárias que receberam o suporte, sugerindo que o endotélio da neoíntima 


\section{Relato de Caso}

é sensível a estímulos químicos, mas pode apresentar resposta paradoxal em alguns casos.

\section{Conclusão}

A reabsorção total do Absorb BVS foi encontrada no seguimento de 5 anos. Após a reabsorção do suporte, parece haver um processo adequado de cicatrização do endotélio vascular, com restauração das propriedades morfológicas e funcionais.

\section{Contribuição dos Autores}

Concepção e desenho da pesquisa: Ramirez LRG, Gutíerrez H, Amat-Santos I; Obtenção de dados: Ramirez LRG, Gutíerrez H, Julca F, Amado M, Varvaro G; Análise e interpretação dos dados: Ramirez LRG, Julca F, Amado M; Redação do manuscrito: Ramirez LRG; Revisão crítica do manuscrito quanto ao conteúdo intelectual importante: Gutíerrez H, Varvaro G, Amat-Santos I.

\section{Potencial Conflito de Interesses}

Declaro não haver conflito de interesses pertinentes.

\section{Fontes de Financiamento}

O presente estudo não teve fontes de financiamento externas.

\section{Vinculação Acadêmica}

Não há vinculação deste estudo a programas de pósgraduação.

\section{Aprovação Ética e Consentimento Informado}

Este estudo foi aprovado pelo Comitê de Ética do Hospital Clinico Universitario de Valladolid sob o número de protocolo PI 18-994. Todos os procedimentos envolvidos nesse estudo estão de acordo com a Declaração de Helsinki de 1975, atualizada em 2013. O consentimento informado foi obtido de todos os participantes incluídos no estudo.

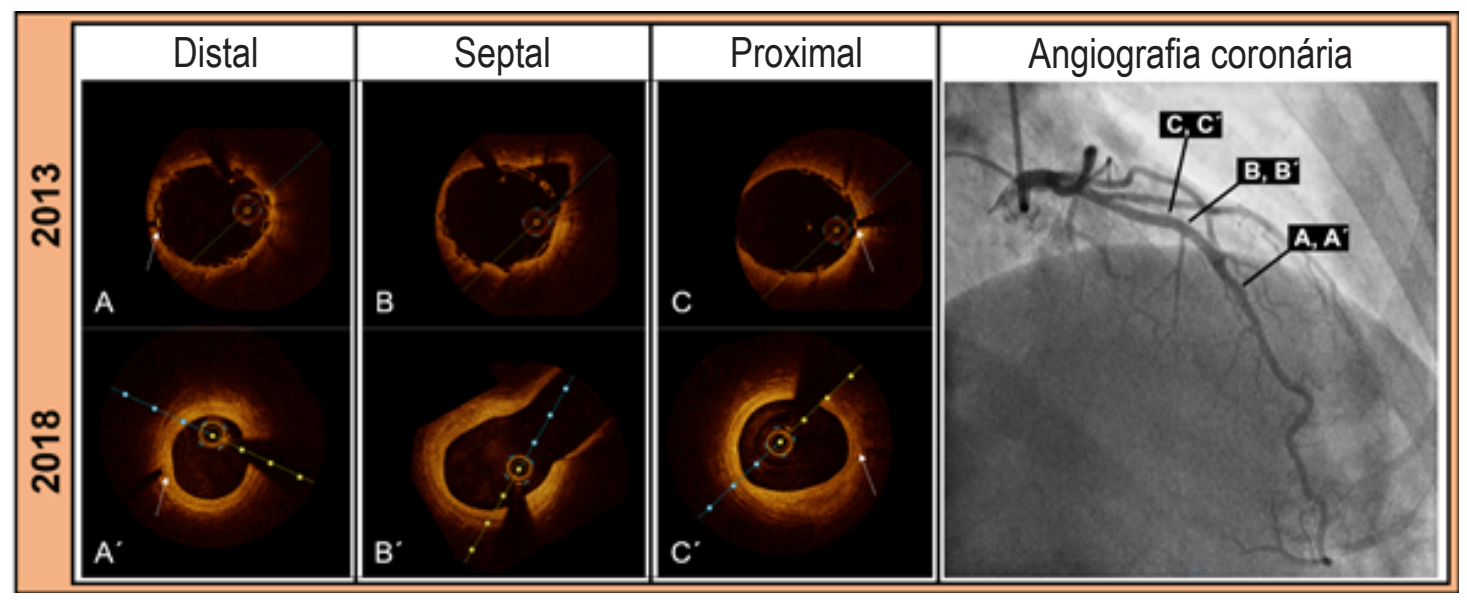

Figura 1 - (A, B, C) Pontos no tempo da implantação do Absorb-BVS por TCO. (A', $\left.B^{\prime}, C^{\prime}\right)$ Achados da TCO no seguimento de 5 anos (mesmo corte transversal). Setas brancas apontam marcadores radiopacos de suportes. 


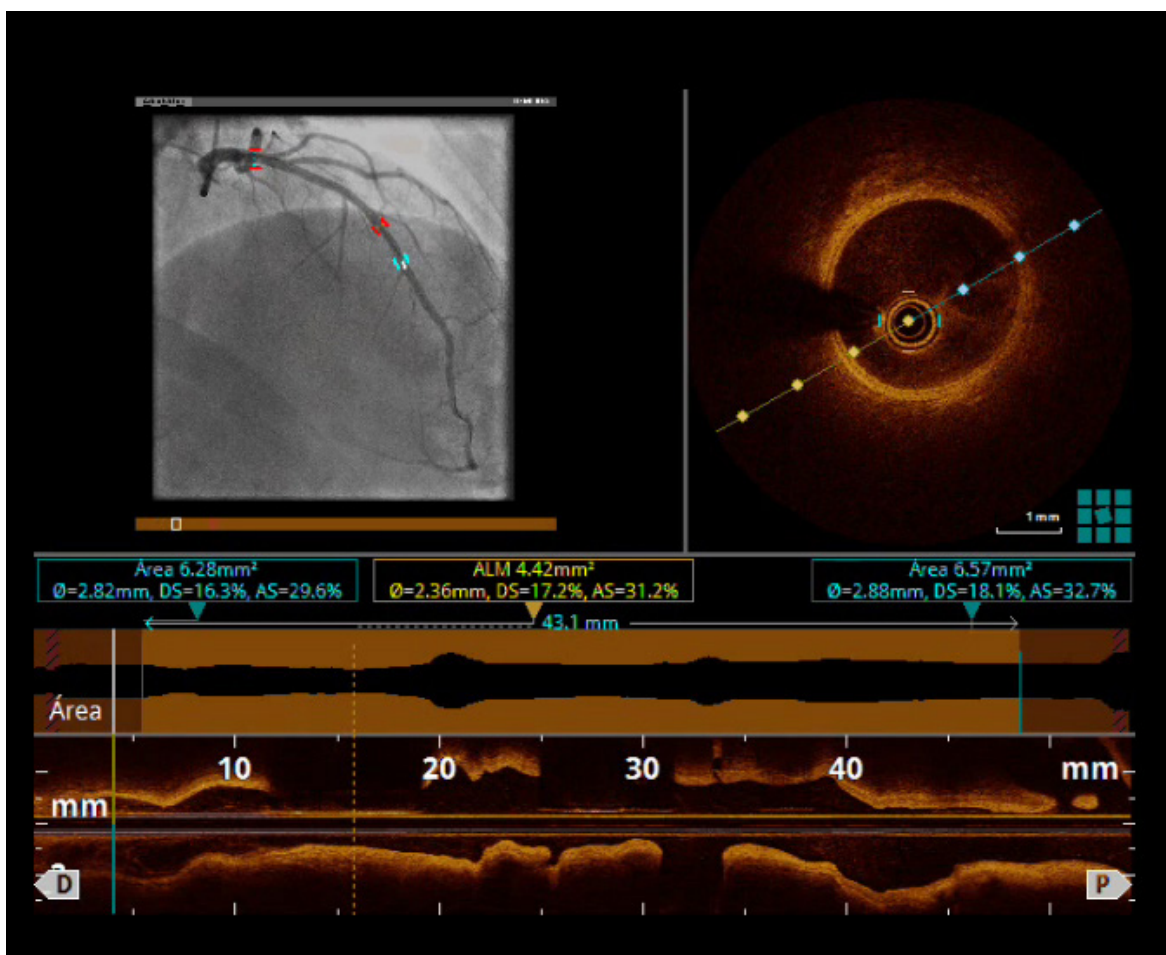

Vídeo 1 - Tomografia de coerência óptica realizada sobre o segmento de andaime de LAD mostrando Absorb BVS totalmente reabsorvido e uma camada neointimal bem organizada. Acesse o vídeo pelo link: http://abccardiol.org/supplementary-material/2021/11601/2019-0783-video1.mp4

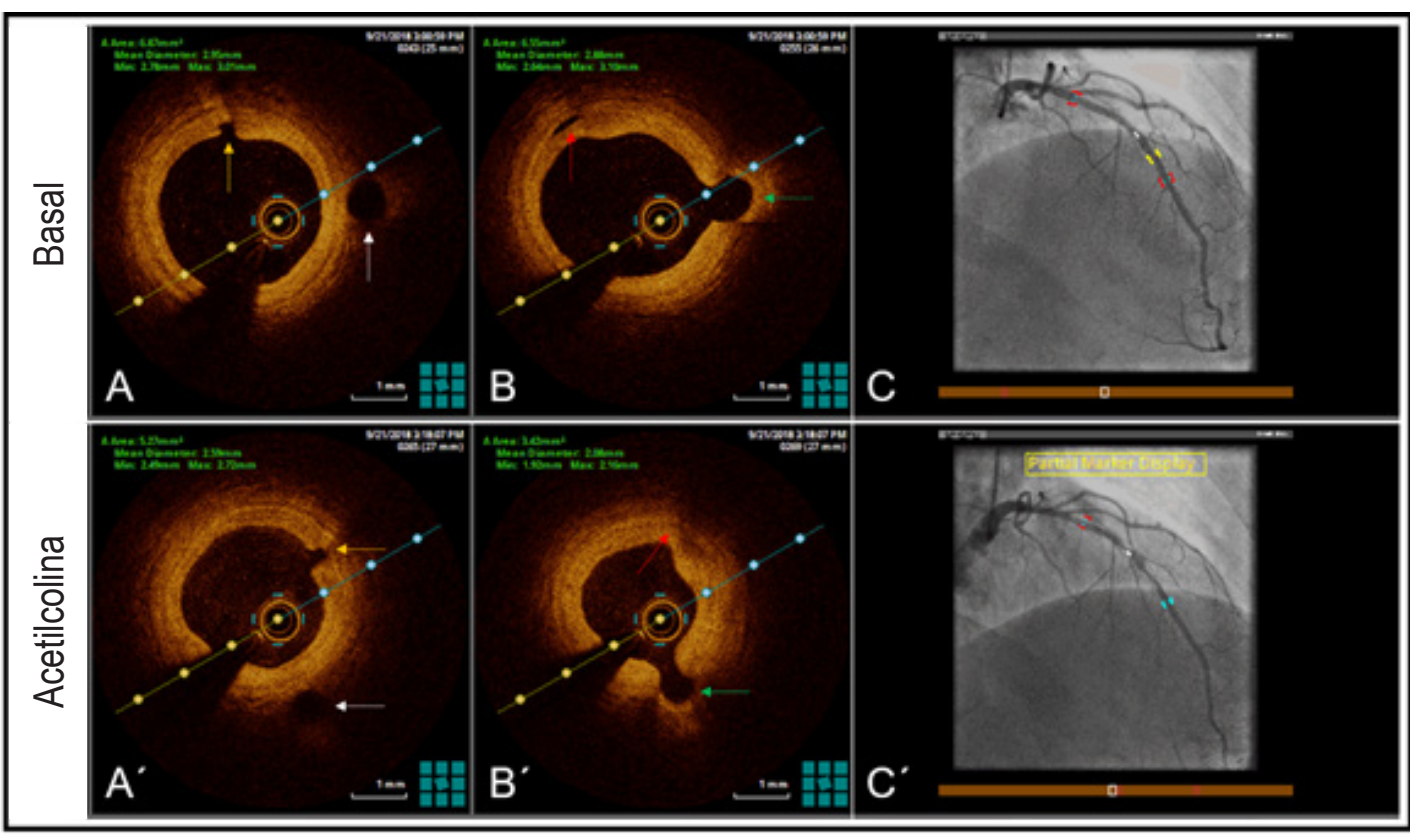

Figura 2 - $(A, B, C)$ Imagens basais obtidas por angiografia e TCO. $\left(A^{\prime}, B^{\prime}, C^{\prime}\right)$ Achados da angiografia e TCO no mesmo corte transversal após dose máxima de acetilcolina. As setas coloridas indicam os ramos laterais antes e depois do teste. 


\section{Relato de Caso}

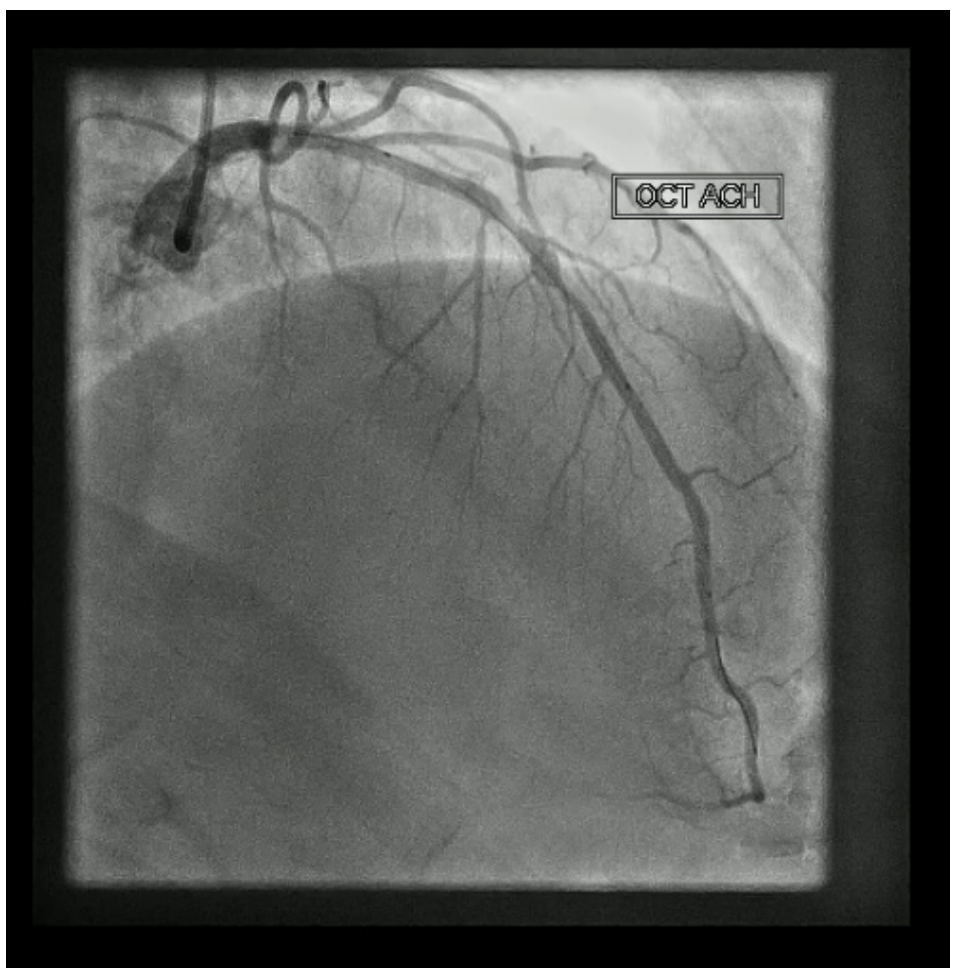

Vídeo 2 - Angiografia coronária mostrando um espasmo LAD -incluindo o segmento de andaime- após o pico da dose de acetilcolina. Acesse o video pelo link: http://abccardiol.org/supplementary-material/2021/11601/2019-0783-video2.mp4

\section{Referências}

1. Ormiston JA, Serruys PW, Regar E, Dudek D, Thuesen L, Webster MW, et al. A bioabsorbable everolimus-eluting coronary stent system for patients with single de-novo coronary artery lesions (ABSORB): a prospective open-label trial. Lancet. 2008;371(9616):899-907.

2. Byrne RA, Stefanini GF, Capodanno D, Onuma Y, Baumbach A, Escaned J, et al. Report of an ESC-EAPCI Task Force on the evaluation and use of bioresorbable scaffolds for percutaneous coronary intervention: executive summary. Eurolntervention. 2018;39(18):1591-601.

3. Serruys PW, Ormiston JA, Onuma Y, Regar E, Gonzalo N, Garcia-Garcia $\mathrm{HM}$, et al. A bioabsorbable everolimus-eluting coronary stent system (ABSORB): 2-year outcomes and results from multiple imaging methods. Lancet. 2009;373(9667):897-910.

4. Onuma Y, Dudek D, Thuesen L, Webster M, Nieman K, Garcia-Garcia $\mathrm{HM}$, et al. Five-year clinical and functional multislice computed tomography angiographic results after coronary implantation of the fully resorbable polymeric everolimus-eluting scaffold in patients with de novo coronary artery disease: the ABSORB cohort A trial. JACC CardiovasC Interv. 2013;6(10):999-1009.

5. Ellis SG, Kereiakes DJ, Metzger DC, Caputo RP, Rizik DG, Teirstein PS, et al. Everolimus-Eluting Bioresorbable Scaffolds for Coronary Artery Disease. N Engl J Med. 2015;373(20):1905-15.

6. Serruys PW, Chevalier B, Dudek D, Cequier A, Carrie D, Iniguez A, et al. A bioresorbable everolimus-eluting scaffold versus a metallic everolimus- eluting stent for ischaemic heart disease caused by de-novo native coronary artery lesions (ABSORB II): an interim 1-year analysis of clinical and procedural secondary outcomes from a randomised controlled trial. Lancet. 2015;385(9962):43-54.

7. Serruys PW, Onuma Y, Garcia-Garcia HM, Muramatsu T, van Geuns RJ, de Bruyne B, et al. Dynamics of vessel wall changes following the implantation of the absorb everolimus-eluting bioresorbable vascular scaffold: a multi-imaging modality study at 6, 12, 24, 36 months. Eurolntervention.2014;9(11):1271-84.

8. Serruys PW, Chevalier B, Sotomi Y, Cequier A, Carrie D, Piek JJ, et al. Comparison of an everolimus-eluting bioresorbable scaffold with an everolimus-eluting metallic stent for the treatment of coronary artery stenosis (ABSORB II): a 3 year, randomised, controlled, single-blind, multicentre clinical trial. Lancet. 2016;388(10059):2479-91.

9. Goncalves-Ramírez LR, Gutiérrez H, Cortés C, Gómez I, San Román JA, Amat-Santos IJ. Functional and structural coronary recovery at the 5-year follow-up after bioresorbable vascular scaffold implantation. An optical coherence tomography analysis. RevEspCardiol.2019;72(4):357-9.

10. Goncalves-Ramírez LR, Gutiérrez H, Rojas P, Cortés C, Serrador A, Ramos B, et al. Fully bioresorption of an Absorb bioresorbable vascular scaffold after scaffold restenosis. Cardiol J.2019;26(2):209-211. 